
3 Research Square
Preprints are preliminary reports that have not undergone peer review.
They should not be considered conclusive, used to inform clinical practice, or referenced by the media as validated information.

\title{
Skin liquid biopsy method for assessing the immune environment of cutaneous T-cell lymphoma lesions
}

\author{
Kan Torii \\ Nagoya City University Graduate School of Medical Sciences \\ Yukinori Okada \\ Osaka University Graduate School of Medicine \\ Akimichi Morita ( $\square$ amorita@med.nagoya-cu.ac.jp) \\ Nagoya City University Graduate School of Medical Sciences
}

\section{Research Article}

Keywords: skin liquid biopsy method, skin inflammatory diseases, cutaneous T-cell lymphoma lesions

Posted Date: April 23rd, 2021

DOI: https://doi.org/10.21203/rs.3.rs-410301/v1

License: (c) (1) This work is licensed under a Creative Commons Attribution 4.0 International License. Read Full License 


\section{Abstract}

(179/200 words) Detailed analysis of cells infiltrating lesional skin cannot be performed in skin biopsy specimens by immunohistochemistry or cell separation techniques because small amounts of protein and minor cell populations in the biopsy specimen might be destroyed by enzyme treatment in the isolation step. Here, we describe a skin liquid biopsy method that enables T cell isolation in small amounts of lesional whole blood from patients with cutaneous T-cell lymphoma. Lesional blood, assumed to contain lesional resident cells, cells from capillary vessels, and blood overflowing from capillary vessels in the lesion area, was obtained during regular skin biopsy. The lesional blood showed substantial increases in distinct cell populations, chemokines, and expression of various genes. CD8 + CD45RO + T cells in the lesional blood negatively correlated with the modified severity-weighted assessment tool scores. CD4 + CD45RO + T cells in the lesional blood expressed genes associated with the development of cancer and progression of cutaneous T-cell lymphoma. The skin liquid biopsy technique might provide new insight into the pathogenesis of mycosis fungoides and facilitate evaluation of the treatment efficacy for other skin inflammatory diseases.

\section{Introduction}

The tissue environment surrounding skin lesions has an important role in skin disease. Harvesting cells from the lesions can be time-consuming and challenging due to considerable cell and protein loss caused by tissue degradation. Various techniques are used to analyze skin lesion cells and the surrounding environment, including multiphoton excitation microscopy [1], dermal open-flow microperfusion [2], and immersion of skin samples in a medium to extract cells [3]. The cell isolation processes required for these techniques, however, may lead to the loss of critical information. For example, while lymphocytes can be isolated from skin tissue obtained by punch biopsy, the small amount of tissue contains too few cells and thus limited information is provided.

Alternatively, lesional blood samples could provide valuable information regarding the surrounding environment, including the levels and types of cytokines and inflammatory cells, without requiring enzyme treatment. In fact, a previous study successfully used sera from the peripheral blood and from blood obtained from psoriasis lesions to assess the skin lesion environment [4]. Therefore, liquid biopsy of skin lesions might be effective for isolating and analyzing cellular components and serum.

Skin biopsies are regularly obtained to diagnose and assess treatment efficacy for cutaneous T-cell lymphoma (CTCL). Diagnosis of CTCL is relatively difficult [5], however, and effective treatments are not yet clearly established. Better methods that allow for rapid isolation and analysis of resident and systemic pathogenic T cells and effector $T$ cells are necessary to facilitate diagnosis and develop effective treatments. Mycosis fungoides (MF), the most common CTCL, is considered to be a low-grade T-cell lymphoma [6]. The premycotic and mycotic phases can last for several years, but in some cases develop very rapidly [7]. Due to the relatively low awareness and diagnostic difficulties of MF, many patients seek dermatologic consultation for the first time after their condition has already progressed to the mycotic or tumor stage. This delay in diagnosis may lead to tumor formation, ulceration, leukemic transformation, visceral invasion, and death within a few months. The histologic findings depend on the disease stage. In the erythema stage (stage I), the characteristic features include epidermal hyperplasia, lymphoid exocytosis, and band-like lymphoid infiltration in the superficial dermis. In the plaque stage (stage II), Pautrier's microabscesses are often observed. In the tumor stage (stage III), tumor cells infiltrate the nodular lesions and proliferate with necrosis causing the subsequent development of ulcers in the tumorous lesions [7]. Cells infiltrating MF lesions have an $\alpha / \beta$ memory T-helper phenotype. In the advanced tumor stage, T-cell markers may be lost and a T-cytotoxic phenotype is observed. Different biopsies of MF lesions obtained from a single patient within a short period of time may reveal several phenotypes [8]. The effector cells that infiltrate MF lesions, however, remain unclear.

A skin liquid biopsy technique could be useful for obtaining more detailed information about the phenotype and immune microenvironment in MF. In the present study, we performed a skin liquid biopsy, i.e., the collection of a small amount of blood from the lesion site during the skin lesion biopsy to determine the types of T cells induced in MF while concurrently analyzing RNA expression. We were also able to determine the functions of the infiltrating $T$ cells with high accuracy. The development of an effective skin liquid biopsy method may also be useful for evaluating other skin inflammatory diseases, such as atopic dermatitis and psoriasis, as well as their treatment efficacies.

\section{Results}

\section{$\mathrm{CD}^{+} / \mathrm{CD} 8^{+} \mathrm{T}$ cells are successfully isolated from a small amount of lesional blood.}

We obtained 200 to $300 \mu \mathrm{L}$ of lesional blood from the wound site resulting from the skin biopsy. The cells were separated from the sample using a cell sorter. Approximately $3000 \mathrm{CD}^{+} \mathrm{T}$ cells and $1000 \mathrm{CD} 8^{+} \mathrm{T}$ cells were successfully isolated from $5 \mu \mathrm{L}$ of peripheral blood (Fig. $1 \mathrm{a}$ ). The number of cells in lesional blood was slightly lower than that in peripheral blood, but we could collect cells from lesional blood (Fig. $1 \mathrm{~b}$ and $\mathrm{c}$ ). Cytometry by time-of-flight revealed that the lesional blood contained more granulocytes, and fewer monocytes and B cells than the peripheral blood (Fig. 1d and e). These findings indicate that the cell population in the lesional blood might differ from that in the peripheral blood. The isolated cells and sera from lesional blood were therefore further analyzed.

$\mathrm{CD}^{+}{ }^{+} \mathrm{CD} 45 \mathrm{RO}{ }^{+}$T-cells in lesional blood negatively correlate with the mSWAT score. 
Fourteen biopsy specimens were obtained from the lesional skin of MF patients and used for immunohistochemical analysis (Table 1). All MF patients were assessed using the modified severity-weighted assessment tool (mSWAT), and skin biopsies were obtained upon admission (Fig. 2a). While the proportions of $\mathrm{CD} 4^{+}$and $\mathrm{CD}^{+} \mathrm{T}$ cells did not differ significantly between lesional blood and peripheral blood in the mass cytometry analysis (Fig. 1e), lesional blood contained significantly a greater proportion of $\mathrm{CD} 4^{+} \mathrm{CD} 45 \mathrm{RO} \mathrm{O}^{+}$and $\mathrm{CD} 8^{+} \mathrm{CD} 45 \mathrm{RO}{ }^{+} \mathrm{T}$ cells (Fig. $2 \mathrm{~b}$ and c).

Furthermore, the proportion of $\mathrm{CD}^{+} \mathrm{CD} 45 \mathrm{RO}^{+} \mathrm{T}$ cells in the lesional and peripheral blood negatively correlated with the mSWAT score (Fig. $2 \mathrm{e}$ ). The proportion of $\mathrm{CD}^{+} \mathrm{CD} 45 \mathrm{RO}^{+} \mathrm{T}$ cells was weakly inversely correlated with the mSWAT score (Fig. 2d). The skin liquid biopsy technique might reveal the phenotypic details of infiltrating cells.

Table 1

Patient background

\begin{tabular}{|c|c|c|c|c|c|c|c|c|c|c|c|c|}
\hline Pt & $\begin{array}{l}\text { Age } \\
(y r)\end{array}$ & Sex & Disease & Stage & mSWAT & $\begin{array}{l}\text { CD8/45RO } \\
(\%, P B)\end{array}$ & $\begin{array}{l}\text { CD8/45RO } \\
(\%, L B)\end{array}$ & $\begin{array}{l}\text { Tissue } \\
\text { CD8/45RO }\end{array}$ & СутоF & RNA-seq & $\begin{array}{l}\text { TCR } \\
\text { repertoire }\end{array}$ & Treatment \\
\hline 1 & 83 & M & MF & IB & 44 & 10.4 & 11.2 & 45 & & & & $\begin{array}{l}\text { Chemo + } \\
\text { PUVA }\end{array}$ \\
\hline 2 & 68 & M & MF & IB & 49 & 0.18 & 0.5 & 45 & & & & Chemo \\
\hline 3 & 61 & $\mathrm{~F}$ & MF & IIIA & 64 & 3.25 & 3.54 & 44 & & & & $\begin{array}{l}\text { Chemo + } \\
\text { PUVA }\end{array}$ \\
\hline 4 & 88 & $\mathrm{~F}$ & MF & IB & 38 & 7.69 & 9.18 & 89 & & CD8/45RO & CD8/45RO & PUVA \\
\hline 5 & 71 & $\mathrm{~F}$ & MF & IB & 102 & 3.12 & 4.03 & 19 & $\bullet$ & & & $\begin{array}{l}\text { Chemo + } \\
\text { PUVA }\end{array}$ \\
\hline 6 & 82 & M & MF & IB & 40 & 0.65 & 0.72 & 67 & 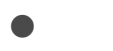 & & & Chemo \\
\hline 7 & 56 & $\mathrm{~F}$ & MF & IB & 36 & 2.22 & 2.33 & 71 & 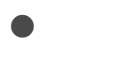 & & & $\begin{array}{l}\text { Chemo + } \\
\text { PUVA }\end{array}$ \\
\hline 8 & 70 & M & MF & IIIA & 138 & 0.34 & 0.26 & 13 & & & & $\begin{array}{l}\text { Chemo + } \\
\text { PUVA }\end{array}$ \\
\hline 9 & 78 & $\mathrm{~F}$ & MF & IB & 22 & 1.71 & 3.81 & 135 & 0 & & & $\begin{array}{l}\text { Chemo + } \\
\text { PUVA }\end{array}$ \\
\hline 10 & 71 & M & MF & IIB & 109 & 0.76 & 0.97 & 23 & $\bullet$ & & CD8/45RO & $\begin{array}{l}\text { Chemo + } \\
\text { PUVA }\end{array}$ \\
\hline 11 & 64 & $\mathrm{~F}$ & MF & IB & 70 & 2.3 & 2.4 & 82 & & & CD4/45RO & $\begin{array}{l}\text { Chemo + } \\
\text { PUVA }\end{array}$ \\
\hline 12 & 72 & $\mathrm{~F}$ & MF & IB & 39 & 12.6 & 11.8 & 167 & & $\mathrm{CD} 4 / 45 \mathrm{RO}$ & & $\begin{array}{l}\text { Chemo + } \\
\text { PUVA }\end{array}$ \\
\hline 13 & 78 & M & MF & IA & 15 & 13 & 15 & 178 & & & & PUVA \\
\hline 14 & 72 & M & MF & IB & 22 & 1.5 & 1.9 & 154 & & & CD8/45RO & $\begin{array}{l}\text { Chemo + } \\
\text { PUVA }\end{array}$ \\
\hline 15 & 45 & $\mathrm{~F}$ & MF & IA & 14 & 1.88 & 2.3 & & & & CD8/45RO & $\begin{array}{l}\text { Chemo + } \\
\text { PUVA }\end{array}$ \\
\hline 16 & 67 & M & MF & IIIA & 94 & 1.16 & 1.81 & & & CD4/45RO & & Chemo \\
\hline 17 & 45 & M & MF & IB & 26 & 1.71 & 1.7 & & & CD4/45RO & & $\begin{array}{l}\text { Chemo + } \\
\text { PUVA }\end{array}$ \\
\hline 18 & 70 & M & MF & IB & 35 & 3.42 & 3.44 & & & CD8/45RO & & $\begin{array}{l}\text { Chemo + } \\
\text { PUVA }\end{array}$ \\
\hline 19 & 34 & $\mathrm{~F}$ & MF & IB & 40 & 1.26 & 1.45 & & & CD8/45RO & & $\begin{array}{l}\text { Chemo + } \\
\text { PUVA }\end{array}$ \\
\hline
\end{tabular}

$\mathrm{CD}^{+}{ }^{+}$CD45RO ${ }^{+}$T-cells infiltrate MF lesions and negatively correlate with CTCL pathogenesis. The CD ${ }^{+}$tumor-infiltrating lymphocyte levels in patients with MF correlate with an improved survival rate and exert an antitumor effect [9]. Therefore, increased levels of CD8 ${ }^{+} \mathrm{T}$ cells in $\mathrm{CTCL}$ serve as a promising criterion for predicting patient survival and supporting treatment decisions and inclusion of patients in randomized controlled trials [10]. Moreover, the partial activation of $\mathrm{CD}^{+}$cytotoxic T cells present in CTCL and their correlation with a better prognosis suggest that they have 
an important role in the antitumor response [11]. Tissue specimens showed a negative correlation between the mSWAT score and $\mathrm{CD} 8^{+} \mathrm{CD} 45 \mathrm{RO}{ }^{+} \mathrm{T}$ cells with less infiltration of effector $C D 8^{+} \mathrm{T}$ cells in advanced cases (Fig. $2 \mathrm{~h}$ and i). In contrast, $\mathrm{CD} 4^{+} \mathrm{CD} 45 \mathrm{RO} \mathrm{O}^{+} \mathrm{T}$ cells in tissue specimens did not correlate with mSWAT (Fig. $2 \mathrm{f}$ and g), consistent with the results from skin liquid biopsy. These infiltrating cells in tissue specimens, however, cannot be easily assessed by only immunohistochemistry.

\section{Chemokine profiles differ between lesional blood and peripheral blood.}

In sera simultaneously isolated from peripheral and lesional blood samples, the levels of chemokines such as CCL5, CCL11, CCL17, CCL22, and CXCL11 were significantly increased in the lesional blood compared with the peripheral blood (Fig. 2j). The increases in these chemokines are specific to MF lesions. CCL5, CCL17, and CCL22 are derived from keratinocytes, CCL22 and CXCL11 are derived from endothelial cells, and CCL11 is derived from macrophages in MF lesions [12]. In particular, levels of CCL17 and CCL22, a CCR4 ligand, are upregulated in the epidermis and serum of patients with MF [13-15]. Malignant T cells expressing CCR4 are recruited by CCL17 and CCL22 [14]. We found that sera from lesional blood revealed a specific chemokine environment for MF. The results demonstrated that the chemokine profile of lesional blood differs from that of peripheral blood, indicating that the skin liquid biopsy technique is feasible for obtaining a detailed chemokine profile of lesional blood.

\section{$\mathrm{CD}^{+} \mathrm{CD} 45 \mathrm{RO}{ }^{+} \mathrm{T}$ cells from lesional and peripheral blood differ in RNA sequence and T-cell receptor repertoire analyses.}

We isolated $\mathrm{CD}^{+} \mathrm{CD} 45 \mathrm{RO}^{+}$and $\mathrm{CD} 8^{+} \mathrm{CD} 45 \mathrm{RO}^{+} \mathrm{T}$ cells from lesional and peripheral blood $(\mathrm{n}=3)$ using a FACS Melody sorter (Becton Dickinson). RNA sequencing (RNA-seq) of the isolated cells was performed to analyze the transcriptome. $\mathrm{CD} 4^{+} \mathrm{CD} 45 \mathrm{RO} \mathrm{O}^{+} \mathrm{T}$ cells in the lesional blood highly expressed genes relating to cancer progression and CTCL pathogenesis: RGS1, RYR2, DNAH9, ANK2, and TNFRSF21 (Fig. 3a) [16-25]. To further confirm the increases in the 51 highly expressed genes, the genes were enriched in the Jensen Disease library of Enrichr. Enrichr showed that the diseases were related to cancer, including "skin cancer" and "lymphoid leukemia" (Fig. 3b). Although we performed T-cell receptor (TCR) repertoire analysis only for 1 case, $\mathrm{CD} 4^{+} \mathrm{CD} 45 \mathrm{RO}^{+} \mathrm{T}$ cells in the lesional blood exhibited a unique TCR repertoire, showing reduced diversity compared with the TCR repertoire in peripheral blood (Fig. 3c and d).

On the other hand, $\mathrm{CD} 8^{+} \mathrm{CD} 45 \mathrm{RO}{ }^{+} \mathrm{T}$ cells highly expressed the following representative genes in lesional blood samples: $C D K N 2 B, P T P R T, C R E B 3 L 1$, CADM4, and INPP5F (Fig. 3e). These genes encode tumor suppressor proteins [26-30] and activate the expression of genes encoding cell cycle inhibitors, including p21 [31]. Gene ontology analysis using 468 genes was performed in $\mathrm{CD}^{+}{ }^{+} \mathrm{CD} 45 \mathrm{RO} \mathrm{O}^{+} \mathrm{T}$ cells from MF samples. Among the 10 most enriched biologic processes determined using Metascape in $\mathrm{CD}^{+} \mathrm{CD} 45 \mathrm{RO}^{+} \mathrm{T}$ cells isolated from MF samples, the most enriched biologic processes were "negative regulation of intracellular signal transduction", "negative regulation of STAT cascade", and "negative regulation for cellular response to growth factor stimulation" (Fig. 3f). A previous study reported that effector cells (CD8 ${ }^{+} \mathrm{CD} 45 \mathrm{RO}{ }^{+} \mathrm{T}$ cells) express exhausted phenotypes [32]. Our findings confirmed that $C D 8^{+} C D 45 R O^{+} T$ cells from lesional blood negatively regulate cellular responses. On the other hand, genes related to inflammation (S100A12, S100A8, HCK, IL 1B and KLF4) were increased in peripheral blood [33-36]. The TCR repertoire of $\mathrm{CD}^{+} \mathrm{CD} 45 \mathrm{RO}^{+} \mathrm{T}$ cells was skewed in lesional blood (erythema area; $\mathrm{n}=4$ ) compared with that in peripheral blood (Fig. $3 \mathrm{~g}$ and $\mathrm{h}$ ). These expanded $\mathrm{CD}^{+} \mathrm{CD} 45 \mathrm{RO}^{+} \mathrm{T}$ cells might be tumor antigen-specific but not have the capacity to suppress CTCL cells.

\section{Stage progression and skewed TCR repertoires in $\mathrm{CD} 8^{+} \mathrm{CD} 45 \mathrm{RO}^{+} \mathrm{T}$ cells.}

Biopsy specimens and lesional blood were collected from each stage: erythema, plaque, and tumor-stage lesions from the same patient. A certain number of $\mathrm{CD}^{+} \mathrm{CD} 45 \mathrm{RO}^{+}$cells were found in the erythema areas, but few were found in the plaque and tumor tissue in case 10 (Fig. $4 \mathrm{a}$, $\mathrm{C}$, e; immunofluorescence staining). We isolated $C D 8^{+} C D 45 R O^{+} T$ cells from the lesional blood and peripheral blood separately. The isolated cells were subjected to TCR repertoire analysis. The $\mathrm{CD} 8^{+} \mathrm{CD} 45 \mathrm{RO} \mathrm{O}^{+} \mathrm{T}$ cells in lesional blood showed unique TCR repertoires in lesions of all stages (Fig. $4 \mathrm{~b}$, d, $\mathrm{f}, \mathrm{g})$. We assume that $\mathrm{CD} 8^{+} \mathrm{CD} 45 \mathrm{RO}^{+}$cells in lesional blood would recognize a tumor antigen in tumor and plaque tissue. In previous reports, malignant T-cell clones exhibit heterogeneity in each skin lesion [37]. Our results are consistent with previous findings that neoplastic T-cell clones vary in skin lesions. Furthermore, different TCR repertoires were present in tumor-stage lesions of the same patient (Fig. 4f), which may result from the generation of different neoplastic T-cell clones for the growth of tumor lesions. In the other patient (case 15), a biopsy was performed from an adjacent lesion, which developed erythema and plaque. $\mathrm{CD} 8^{+} \mathrm{CD} 45 \mathrm{RO} \mathrm{O}^{+}$cells were found in both erythema and plaque lesions (Fig. $4 \mathrm{~h}$ and $\mathrm{j}$ ), and repertoire analysis showed that the same TCRs (TRAV1-2 - TRAJ33) were increased (Fig. 4i, k, I). These results indicate CD ${ }^{+}{ }^{T}$ cells with different TCR repertoires are directed for each skin lesion, which is expected given the heterogeneity of malignant T cells in skin lesions.

\section{Discussion}

The present study revealed that the composition of lesional blood differs from that of peripheral blood, and lesional blood appears to reflect the condition of the skin's immune environment of lesional areas in MF patients. Many techniques are used to analyze the cells and tissue environments of skin lesions, and the skin liquid biopsy method is a feasible technique for assessing lesion areas. Although the skin liquid biopsy method would not obviate the need for skin biopsy due to the different results, analysis of lesional blood could provide valuable information about 
infiltrating cells and the separated serum. We assume that lesional blood would contain lesional resident cells, cells from capillary vessels, and blood overflowing from capillary vessels, allowing us to identify a unique pattern of cell populations and chemokines in the lesional blood.

Mass cytometry analysis showed differences in the cell populations, and the chemokine assay revealed increases in specific chemokines in lesional blood. The different types of cells in the lesional blood may be due to the induction of chemokines. Memory $\mathrm{T}^{\text {cells, }} \mathrm{CD} 45 \mathrm{RO}{ }^{+} \mathrm{T}$ cells, were frequently obtained in lesional blood, suggesting that these cells are involved in the lesion. The chemokines, which are reported to be associated with MF, were particularly abundant in lesional blood. CCL17 and CCL22 are ligands for CCR4, and these chemokines are strongly associated with the pathogenesis of MF $[13,14]$. Although we have not yet collected lesional blood from healthy areas, we will be able to clarify the pathogenesis of MF in detail by comparing lesional blood from healthy and lesional areas.

RNA-seq of $\mathrm{CD}^{+} \mathrm{CD} 45 \mathrm{RO}^{+} \mathrm{T}$ cells and $\mathrm{CD} 8^{+} \mathrm{CD} 45 \mathrm{RO}^{+} \mathrm{T}$ cells suggested that lesional blood might contain malignant $\mathrm{T}$ cells and effector $\mathrm{T}$ cells, respectively. This notion was supported by the skewed TCR repertoire in lesional blood (Fig. $3 \mathrm{c}$ and g). In the present study, $\mathrm{CD} 4^{+} \mathrm{CD} 45 \mathrm{RO}{ }^{+} \mathrm{T}$ cells from the lesional blood highly expressed genes relating to cancer progression and CTCL progression. It may thus be possible to isolate lymphocytes that are recruited by chemokines or present in the lesion area from lesional blood. A larger number of differentially expressed genes were detected in the $\mathrm{CD} 4^{+} \mathrm{CD} 45 \mathrm{RO}^{+}$cells of the peripheral blood because the cells of lesional blood have individual variations. In addition, there are different types of malignant cells [38], which may be why fewer differentially expressed genes were detected in the CD $4^{+} \mathrm{CD} 45 \mathrm{RO}{ }^{+}$cells of lesional blood (Fig. 3a). $\mathrm{CD}^{+} \mathrm{CD} 45 \mathrm{RO}^{+} \mathrm{T}$ cells in the lesional blood had a less inflammatory transcriptome than the $\mathrm{CD} 8^{+} \mathrm{CD} 45 \mathrm{RO} \mathrm{O}^{+} \mathrm{T}$ cells in the peripheral blood. Effector $\mathrm{T}$ cells express exhausted phenotypes characterized by the expression of the PD-1, ICOS, TIM-3, LAG-3, and CTLA-4 markers in lesional skin [32]. Although the expression of these genes was not increased, the weak expression of genes of the inflammatory system and cell division suggest that $\mathrm{CD}^{+} \mathrm{CD} 45 \mathrm{RO}^{+} \mathrm{T}$ cells in the lesional blood are a type of exhausted $\mathrm{T}$ cells [39].

The clones of malignant cells are different in skin lesions in MF [40]. In the present study, we collected $\mathrm{CD} 8^{+} \mathrm{CD} 45 \mathrm{RO} \mathrm{O}^{+}$cells of lesional blood and performed TCR repertoire analysis by skin lesion. Few $C D 8^{+} C D 45 R O^{+}$cells, however, were detected in the plaque and tumor tissue in case 10 . A characteristic repertoire pattern of $\mathrm{CD} 8^{+} \mathrm{CD} 45 \mathrm{RO}^{+}$cells was detected in different skin lesions of case 10.

Case 15 showed a more skewed repertoire of the plaque tissue compared with an area of erythema from the same skin lesion. Although the lesional blood would not be a perfect representation of the cells in this lesion, we could indirectly show that different skin lesions have different clones of malignant cells. We need to further increase the number of cases and investigate the details in the future.

In conclusion, a skin liquid biopsy method for assessing the immune environment of CTCL was successfully developed in this study. Sufficient numbers of isolated cells and amounts of sera were successfully obtained from a small amount of lesional blood collected by skin liquid biopsy. The cells and sera isolated from lesional blood can also be used for further gene expression analysis of target cells. Our results might provide new insight into the pathogenesis of MF, a rare cutaneous lymphoma. The technique described in this study could be further applied to evaluate other skin inflammatory diseases, such as atopic dermatitis and psoriasis, as well as for assessing the efficacy of treatments for these diseases.

\section{Methods}

Patients. We recruited 19 patients with MF (mean age: $67.10 \pm 13.91$ years; 9 women, 10 men) from the Department of Dermatology at the Nagoya City University. Exclusion criteria were: 1) age under 20 years, 2) HTLV-1 positive status, and 3) pregnant. The institutional review board of the Nagoya City University Graduate School of Medical Sciences approved the study (approval number: \#60-18-0101). Written informed consent was obtained from the patients. All the experimental protocols adhered to relevant ethical guidelines for involving humans. The patients' profiles are described in Table 1. Samples were obtained from the first 14 patients who visited our department from December 2018 to December 2019 and used for tissue staining, flow cytometry analyses, and chemokine assays. Some samples were subjected to RNA-seq (cases 4 and 12) and TCR repertoire analyses (cases $4,10,11$, and 14). Samples obtained from cases 15 through 19 were used for RNA-seq or TCR repertoire analyses (Table 1).

Lesional blood collection. Skin biopsies were regularly obtained from the patients for diagnosis and assessment of treatment efficacy. After administering local anesthesia using lidocaine without epinephrine to the skin lesion, a punch biopsy was performed at a depth of 1- to 3-mm to avoid reaching the fatty layer. Oozing blood from the wounded area was collected as quickly as possible into an Eppendorf tube containing anticoagulant to avoid clotting. As anticoagulants, we used $5 \mu \mathrm{L}$ of $100 \mathrm{U} \mathrm{mL}^{-1}$ heparin sodium (TERUMO) for flow cytometry analysis, and $3 \mu \mathrm{L}$ of 0.5 mol L ${ }^{-1}$ EDTA (Invitrogen) for the RNA-seq and TCR repertoire analyses. Approximately 200 to $300 \mu \mathrm{L}$ of blood was collected using a P20 Pipetman (Gilson) from each lesion area. We also collected 30 to $50 \mu \mathrm{L}$ of lesional blood without anticoagulant to obtain serum. After collecting the lesional blood, we performed another punch biopsy in the same lesion area, again at a sufficient depth to obtain a skin sample. Serum was collected and stored at $-80^{\circ} \mathrm{C}$ until analysis. We collected peripheral blood from the patient's arm and treated the peripheral blood in the same manner as the lesional blood.

Flow cytometry. Peripheral and lesional blood samples were stained with antibodies specific for CD3 (clone SK7), CD4 (clone SK3), CD8 (clone SK1), CD45RA (clone L48), and CD45RO (clone UCHL-1) for 20 min at 23-25 ${ }^{\circ}$. All antibodies used for flow cytometry were purchased from BD 
Biosciences. The antibodies for peripheral and lesional blood staining were hemolyzed with BD PharmLyse (BD Biosciences) for 15 min at room temperature, centrifuged, and then resuspended in staining buffer (BD Biosciences). Cells were analyzed on a fluorescence-activated cell sorting (FACS) Verse flow cytometer (BD Biosciences), and data analysis was performed using the FlowJo software (FlowJo, version 10.6.2).

Chemokine analysis. Serum levels of CCL2, CCL3, CCL4, CCL5, CCL11, CCL17, CCL20, CXCL5, CXCL8, CXCL9, CXCL10, and CXCL11 were determined using the Human Proinflammatory Chemokine Panel (BioLegend), and serum levels of CCL21 and CCL22 were determined using the AimPlex Premixed Multiplex kit (AimPlex Biosciences) according to the manufacturer's instructions. All samples were read on a FACS Verse (BD Biosciences).

Mass cytometric immunoassay. The blood cells were stained for mass cytometry after hemolysis using a Maxpar Human Peripheral Blood Phenotyping Panel kit (Fluidigm). Hemolyzed peripheral blood and lesional blood samples were resuspended in $1 \mathrm{~mL}$ phosphate-buffered saline and incubated for $5 \mathrm{~min}$ at room temperature with $1 \mathrm{~mL}$ of Cisplatin-108Pt (Fluidigm). The cells were washed using Maxpar Cell Staining Buffer (Fluidigm) and centrifuged, and the supernatant discarded; the pellets were resuspended in $50 \mu \mathrm{L}$ of the same buffer. We then added $50 \mu \mathrm{L}$ of a prepared cocktail of titrated Maxpar metal-conjugated antibodies (Fluidigm). After incubating for 15 min at room temperature, we washed the cells twice and fixed them with $2 \%$ paraformaldehyde. The stained cells were analyzed by St. Luke's MBL Corp using cytometry by time-of-flight. Mass cytometry data were analyzed using Cytobank (https://www.cytobank.org/).

Cell sorting. $\mathrm{CD}^{+} \mathrm{CD} 45 \mathrm{RO}^{+}$and $\mathrm{CD} 8^{+} \mathrm{CD} 45 \mathrm{RO} \mathrm{O}^{+} \mathrm{T}$ cells were sorted using the BD FACSMelody Cell Sorter (BD Bioscience). The sorted T cells were collected for the TCR repertoire and RNA-seq analyses as described below.

RNA-seq analysis. The $\mathrm{CD} 4^{+} \mathrm{CD} 45 \mathrm{RO}^{+}$and $\mathrm{CD} 8^{+} \mathrm{CD} 45 \mathrm{RO} \mathrm{O}^{+} \mathrm{T}$ cells were prepared as described above. The $\mathrm{T}$ cells were lysed with $\mathrm{TRIzol}$ reagent (Thermo Fisher Scientific) and stored at $-80^{\circ} \mathrm{C}$. The lysates were sent to Genewiz Japan Corp for RNA-sEq. RNA-seq and related analyses were completed by Genewiz Japan Corp. In brief, RNA was extracted with chloroform/isopropanol and recovered from the supernatants using RNA Clean and Concentrator-5 columns (ZymoResearch) following the manufacturer's instructions. The RNA purity was assessed with an Agilent 2100 Bioanalyzer. The RNA was subjected to library preparation with the TaKaRa SmartSeq Stranded Kit (Takara Bio) and sequenced with Illumina Hiseq (Illumina). Sequences were mapped to grch38 with HISAT2 (version 2.0.1). Differentially expressed genes were counted using the DESeq2 package in R (version 3.6.3). Up- and downregulated genes were defined as those (i) differentially expressed in peripheral and lesional blood cells with a $P$ value lower than $\mathbf{0 . 0 5}$, and (ii) having a greater than 2 -fold change in the average normalized number of peripheral and lesional blood cells. Gene ontology analysis and enrichment analysis using the Jensen DISEASES dataset of differentially expressed genes was performed using the Enrichr webtool (https://maayanlab.cloud/Enrichr/) and Metascape (https://metascape.org/) [41-43].

TCR repertoire analysis. $\mathrm{CD} 4^{+} \mathrm{CD} 45 \mathrm{RO} \mathrm{O}^{+}$and $\mathrm{CD} 8^{+} \mathrm{CD} 45 \mathrm{RO} \mathrm{O}^{+} \mathrm{T}$ cells were prepared as described above. The $\mathrm{T}$ cells were lysed with Isogen-LS (NIPPON GENE) and stored at $-80^{\circ} \mathrm{C}$. The lysates were sent to Repertoire Genesis Inc. (Ibaraki, Japan) for next-generation sequencing, which was performed as previously described [44]. Briefly, total RNA was converted to complementary DNA (cDNA) with the SuperScript reverse transcriptase (Invitrogen). Double-stranded -cDNA was synthesized and ligated with a 5 ' adaptor oligonucleotide, then cut with the Sphl restriction enzyme. Next, the double stranded-cDNA was amplified through polymerase chain reaction using primers specific for the adaptor and TCRa constant region. The sequencing was performed with the Illumina MiSeq paired-end platform ( $2 \times 300 \mathrm{bp})$. Data processing was performed with the repertoire analysis software developed by Repertoire Genesis Inc. TCR sequences were assigned with a dataset of reference sequences from the international ImMunoGeneTics information system database (http://www.imgt.org). The Shannon-Weaver index shows the diversity and is defined as follows.

Shannon-Weaver index $=-\sum_{i-1}^{S}\left(\frac{n_{i}}{N} \ln \frac{n_{i}}{N}\right)$

Immunohistochemistry and immunofluorescence staining. Biopsy skin specimens were fixed in $10 \%$ formalin and embedded in paraffin. Skin specimens were cut from the tissue block in 4- $\mu \mathrm{m}$ sections and stained with hematoxylin and eosin, anti-CD4 antibodies (Ab; clone EPR6855, Abcam), anti-CD8 Ab (clone C8/144B, Abcam), or anti-CD45RO Ab (clone UCH-L1, Absolute Antibody). Alexa488-conjugated goat anti-mouse IgG Ab (Invitrogen) and Alexa594-conjugated goat anti-rabbit IgG Ab (Invitrogen) were used as secondary antibodies. Fluorescent images were obtained using BZ-X810 (Keyence). The number of cells was determined with the cell count software BZ-H4C (Keyence).

Statistics. Statistical analyses were performed using GraphPad Prism 7. All numerical data are summarized using mean \pm standard deviation. Paired or unpaired Student t-tests were used to determine the significance of differences between groups, unless otherwise indicated in the figure legend. P-values $<0.05$ were considered statistically significant.

\section{Declarations}

\section{Acknowledgments}


We thank Akiko Nishioka and Saori Kasuya for technical assistance; Kyoko Ikumi, Emi Nishida, Tetsuya Magara, and Mai Sakurai for clinical sampling; and Motoki Nakamura for tissue staining.

\section{Author contributions}

A. Morita conceived the study. A. Morita and K. Torii designed the experiments, analyzed the data, and wrote the manuscript. K. Torii performed the experiments. Y. Okada analyzed the data and wrote the manuscript.

\section{Funding}

This work was supported by a Grant-in-Aid for Scientific Research B $20 \mathrm{H} 03703$ (AM) from the Japan Society for the Promotion of Science. The funding agency played no role in the study design, data collection or analysis, the decision to publish, or preparation of the manuscript.

\section{Competing interests}

The authors declare no competing financial interests.

\section{Data availability}

All RNA-seq data sets have been deposited in the DNA Data Bank of Japan under accession numbers DRA010717 (http://www.ddbj.nig.ac.jp/introe.html). All data are available from the corresponding author upon reasonable request.

\section{References}

1. Li, J.L. et al. Intravital multiphoton imaging of immune responses in the mouse ear skin. Nat. Protoc. 7, 221-234 (2012).

2. Bodenlenz, M. et al. Clinical applicability of dOFM devices for dermal sampling. Skin Res. Technol. 19, 474-483 (2013).

3. Hashizume, H. et al. In vitro propagation and dynamics of T cells from skin biopsies by methods using interleukins-2 and -4 or anti-CD3/CD28 antibody-coated microbeads. Acta Derm. Venereol. 90, 468-473 (2010).

4. Ishimoto, T., Kataoka, S., Shiga, T., Takaishi, M. \& Sano, S. Use of intralesional blood to determine diffusible biomarkers from skin lesions. J. Dermatol. Sci. 90, 75-81 (2018).

5. Weed, J. \& Girardi, M. The difficult-and often delayed-diagnosis of CTCL. Sci. Transl. Med. 7, $308 \mathrm{fs} 41$ (2015).

6. Jawed, S.I., Myskowski, P.L., Horwitz, S., Moskowitz, A. \& Querfeld, C. Primary cutaneous T-cell lymphoma (mycosis fungoides and Sézary syndrome): part I. Diagnosis: clinical and histopathologic features and new molecular and biologic markers. J. Am. Acad. Dermatol. 70, 205.e1-16 (2014).

7. Abel, E.A., Wood, G.S. \& Hoppe, R.T. Mycosis fungoides: clinical and histologic features, staging, evaluation, and approach to treatment. $C A$ Cancer J. Clin.43, 93-115 (1993).

8. Kash, N., Massone, C., Fink-Puches, R. \& Cerroni, L. Phenotypic variation in different lesions of mycosis fungoides biopsied within a short period of time from the same patient. Am. J. Dermatopathol. 38, 541-545 (2016).

9. Hoppe, R.T., Medeiros, L.J., Warnke, R.A. \& Wood, G.S. CD8-positive tumor-infiltrating lymphocytes influence the long-term survival of patients with mycosis fungoides. J. Am. Acad. Dermatol. 32, 448-453 (1995).

10. Abeni, D. et al. Circulating CD8+ lymphocytes, white blood cells, and survival in patients with mycosis fungoides. Br. J. Dermatol. 153, 324-330 (2005).

11. Vermeer, M.H. et al. CD8+ T cells in cutaneous T-cell lymphoma: expression of cytotoxic proteins, Fas Ligand, and killing inhibitory receptors and their relationship with clinical behavior. J. Clin. Oncol. 19, 4322-4329 (2001).

12. Wu, X.S., Lonsdorf, A.S. \& Hwang, S.T. Cutaneous T-cell lymphoma: roles for chemokines and chemokine receptors. J. Invest. Dermatol. 129, 1115-1119 (2009).

13. Kakinuma, T. et al. Thymus and activation-regulated chemokine (TARC/CCL17) in mycosis fungoides: serum TARC levels reflect the disease activity of mycosis fungoides. J. Am. Acad. Dermatol. 48, 23-30 (2003).

14. Kayo, T. et al. Bexarotene reduces production of CCL22 from tumor-associated macrophages in cutaneous T-cell lymphoma. Front. Oncol. $\mathbf{9}$, 907 (2009), doi: 10.3389/fonc.2019.00907.

15. Ferenczi, K., Fuhlbrigge R.C., Pinkus, J.L., Pinkus, G.S. \& Kupper, T.S. Increased CCR4 expression in cutaneous T cell lymphoma. J. Invest. Dermatol. 119, 1405-1410 (2002).

16. Laharanne, E. et al. Genome-wide analysis of cutaneous T-cell lymphomas identifies three clinically relevant classes. J. Invest. Dermatol. 130, 1707-1718 (2010).

17. Hurst, J.H. \& Hooks, S.B. Regulator of G-protein signaling (RGS) proteins in cancer biology. Biochem. Pharmacol. 78, 1289-1297 (2009).

18. Mariot, P. et al. Evidence of functional ryanodine receptor in apoptosis of prostate cancer (LNCaP) cells. Prostate 43, 205-214 (2000). 
19. Abdul, M., Ramlal, S. \& Hoosein, N. Ryanodine receptor expression correlates with tumor grade in breast cancer. Pathol. Oncol. Res. 14, 157160 (2008).

20. Nairismägi, M.L. et al. JAK-STAT and G-protein-coupled receptor signaling pathways are frequently altered in epitheliotropic intestinal T-cell lymphoma. Leukemia 30, 1311-1319 (2016).

21. Marschalkó, M. et al. Folliculotropic mycosis fungoides: Clinicopathological analysis of 17 patients. J. Eur. Acad. Dermatol. Venereol. 29, 964972 (2015).

22. Budinska, E. et al. Gene expression patterns unveil a new level of molecular heterogeneity in colorectal cancer. J. Pathol. 231, 63-76 (2013).

23. Iqbal, J. et al. Gene expression signatures delineate biological and prognostic subgroups in peripheral T-cell lymphoma. Blood 123, 2915-2923 (2014).

24. Shin, J. Lesional gene expression profiling in cutaneous T-cell lymphoma reveals natural clusters associated with disease outcome. Blood $\mathbf{1 1 0}$ (3015-3027) (2007).

25. Huang, Y. et al. Gene expression profiling identifies emerging oncogenic pathways operating in extranodal NK/T-cell lymphoma, nasal type. Blood 115, 1226-1237 (2010).

26. Jafri, M. et al. Germline mutations in the CDKN2B tumor suppressor gene predispose to renal cell carcinoma. Cancer Discov. 5, 723-729 (2015).

27. Scott, A. \& Wang, Z. Tumour suppressor function of protein tyrosine phosphatase receptor-T. Biosci. Rep. 31, 303-307 (2011).

28. Jang, S.M. et al. Clinicopathological significance of CADM4 expression, and its correlation with expression of E-cadherin and Ki-67 in colorectal adenocarcinomas. J. Clin. Pathol. 65, 902-906 (2012).

29. Luo, F., Zhao, Y. \& Liu, J. Cell adhesion molecule 4 suppresses cell growth and metastasis by inhibiting the Akt signaling pathway in non-small cell lung cancer. Int. J. Biochem. Cell Biol. 123, 105750 (2020).

30. Kim, H.S., Li, A., Ahn, S., Song, H. \& Zhang, W. Inositol polyphosphate-5-phosphatase F (INPP5F) inhibits STAT3 activity and suppresses gliomas tumorigenicity. Sci. Rep. 4, 7330 (2014).

31. Denard, B., Lee, C. \& Ye, J. Doxorubicin blocks proliferation of cancer cells through proteolytic activation of CREB3L1. Elife 1, 00090 (2012), doi:10.7554/eLife.00090.

32. Querfeld, C. et al. Primary T cells from cutaneous T-cell lymphoma skin explants display an exhausted immune checkpoint profile. Cancer Immunol. Res. 6, 900-909 (2018).

33. Yang, Z. et al. Proinflammatory properties of the human S100 protein S100A12. J. Leukoc. Biol. 69, 986-94 (2001).

34. Roseweir, A.K. et al. Src family kinases, HCK and FGR, associate with local inflammation and tumour progression in colorectal cancer. Cell. Signal. 56, 15-22 (2019).

35. Henao-Mejia, J., Elinav, E., Strowig, T. \& Flavell, R.A. Inflammasomes: Far beyond inflammation. Nat. Immunol. 13, 321-324 (2012).

36. Shaverdashvili, K. et al. KLF4 activates NFKB signaling and esophageal epithelial inflammation via the Rho-related GTP-binding protein RHOF. PLoS One 14, e0215746 (2019).

37. lyer, A. et al. Skin colonization by circulating neoplastic clones in cutaneous T-cell lymphoma. Blood 134, 1517-1527 (2019).

38. Murray, D. et al. Progression of mycosis fungoides occurs through divergence of tumor immunophenotype by differential expression of HLADR. Blood Adv. 3, 519-530 (2019).

39. Kallies, A., Zehn, D. \& Utzchneider, D.T. Precursor exhausted T cells: key to successful immunotherapy? Nat. Rev. Immunol. 20, 128-136 (2020).

40. Lyer, A. et al. Branched evolution and genomic intratumor heterogeneity in the pathogenesis of cutaneous T-cell lymphoma. Blood Adv. 4, 2489-2500 (2020).

41. Pletscher-Frankild, S., Pallejà, A., Tsafou, K., Binder, J.X. \& Jensen, L.J. DISEASES: Text mining and data integration of disease-gene associations. Methods 74, 83-89 (2015).

42. Chen, E.Y. et al. Enrichr: interactive and collaborative HTML5 gene list enrichment analysis tool. BMC Bioinformatics. 14, 128 (2013).

43. Zhou, Y. et al. Metascape provides a biologist-oriented resource for the analysis of systems-level datasets. Nat. Commun. 10, 1523 (2019).

44. Yoshida, R. et al. A new method for quantitative analysis of the mouse T-cell receptor $V$ region repertoires: comparison of repertoires among strains. Immunogenetics 52, 35-45 (2000).

\section{Figures}


a

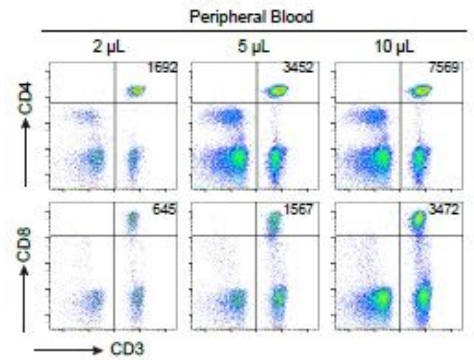

b

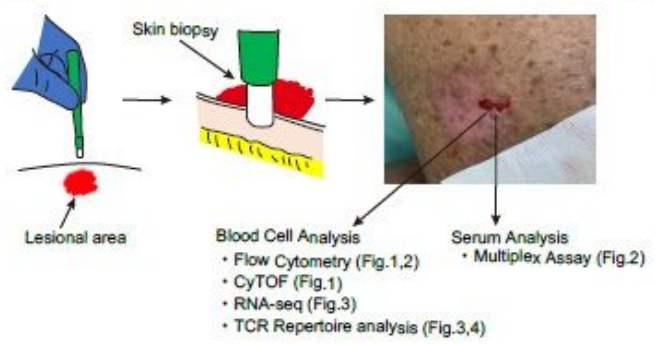

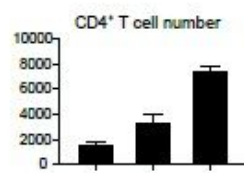
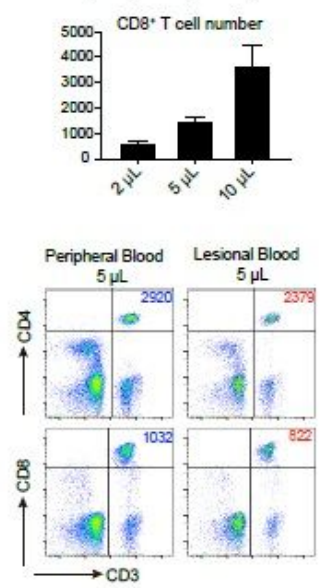

CD4. $T$ cell number

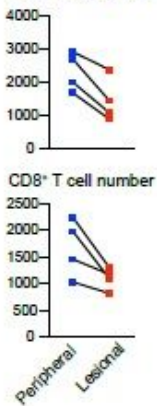

d
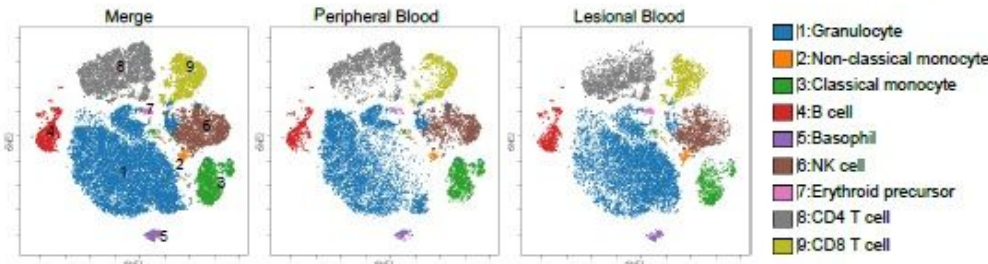

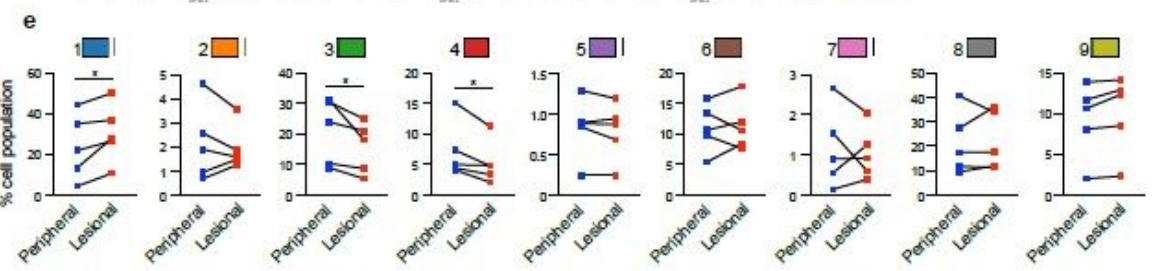

Figure 1

Cell populations differ between peripheral and lesional blood in patients with mycosis fungoides (MF). a) Flow cytometry analysis of CD4+ and CD8+ T cells from 2, 5 and $10 \mathrm{~mL}$ peripheral blood for the detection of $\mathrm{T}$ cells in small amounts of blood. Number in the upper right corner represents the number of CD4+ and CD8+ T cells. Mean (+ standard deviation) numbers of CD4+ and CD $8+$ cells ( $n=3)$ are shown in the bar graph (right). b) Lesional blood was collected during a skin biopsy. Peripheral and lesional blood was obtained from the patient's arm and erythematous lesion, respectively, on the same day. The collected blood cells and serum were used for the subsequent experiments. c) Flow cytometry of CD4+ and CD8+ T cells obtained from $5 \mathrm{~mL}$ of peripheral and lesional blood. Numbers of CD4+ and CD8+ T cells in $5 \mathrm{~mL}$ of peripheral and lesional blood are shown (right, $n=4$ ). d) Representative mass cytometry analysis of peripheral and lesional blood from a patient with MF through viSNE analysis using Cytobank. The color of each dot represents its immune cell subset. e) The proportion of each cell population in peripheral and lesional blood obtained from patients with MF $(n=5)$ quantified by mass cytometry. A paired t-test was used for statistical analysis. * $P<0.05$. 


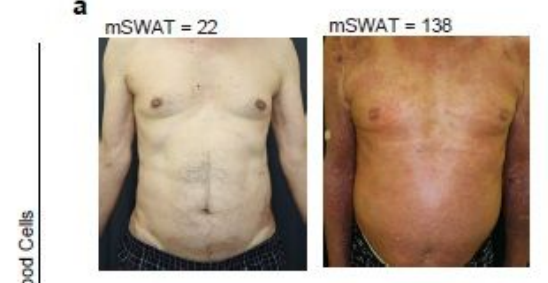

d

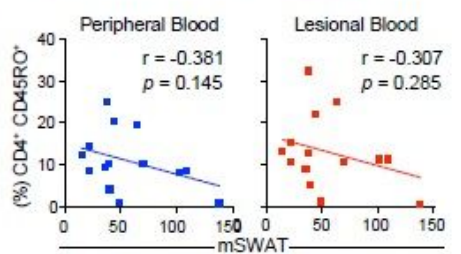

b

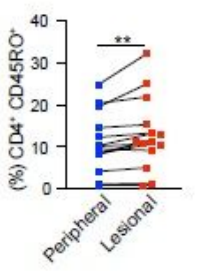

c

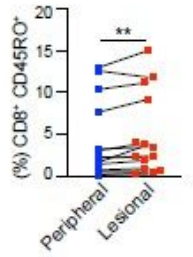

e

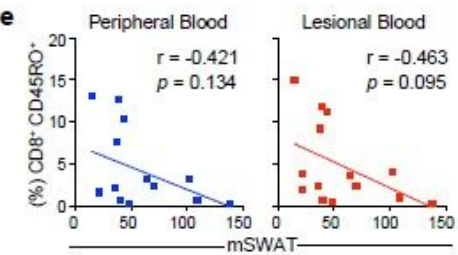

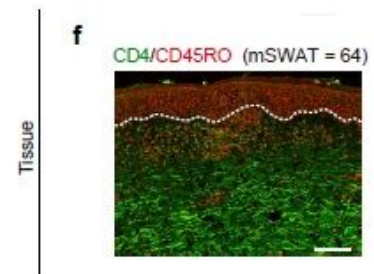

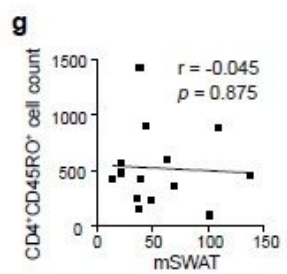

h

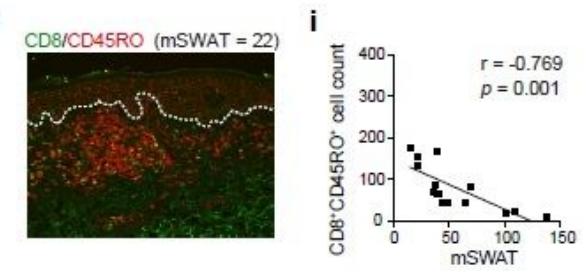

j
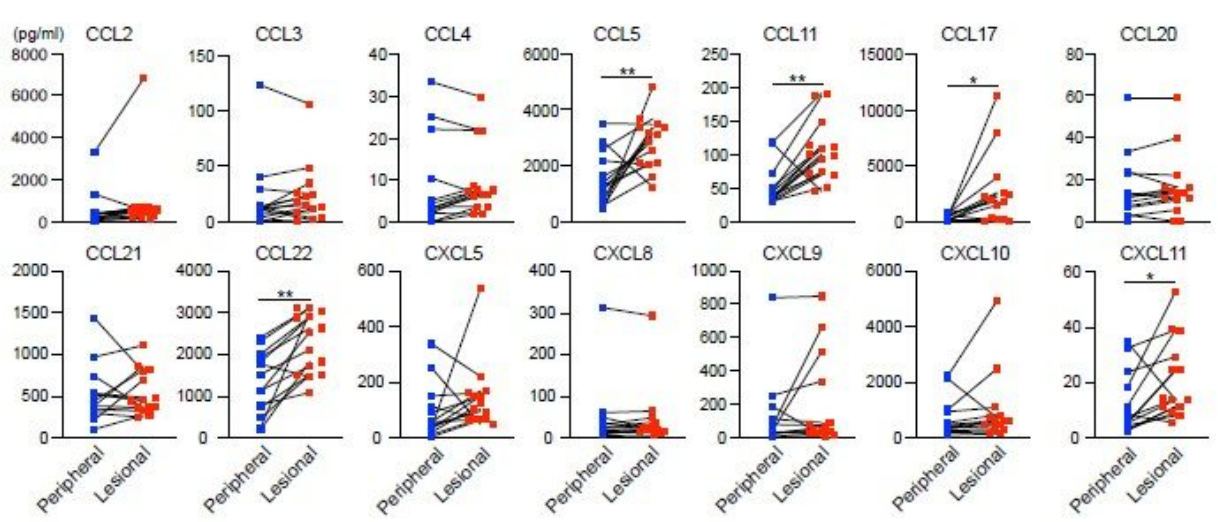

Figure 2

CD8+CD45RO+ cells negatively correlate with the mSWAT score in patients with MF. a) Typical clinical features of MF lesional skin with an mSWAT score of 22 (left) and an mSWAT score of 138 (right). b, c) Percentage of CD4+CD45RO+ and CD8+CD45RO+ T cells in lesional blood is higher than that that of peripheral blood. Paired t-test. $* * P<0.01$. $d, e)$ Pearson correlation coefficient analysis for the correlation of CD4+CD45RO+ $T$ cells and CD8+CD45RO+ T cells in peripheral blood and lesional blood with mSWAT $(n=14) . f-i)$ Representative immunofluorescence staining of CD4/8 (green) and CD45RO (red) in MF lesional skin. Scale bar = $100 \mathrm{~m}$ Pearson correlation coefficient analysis for the correlation of CD4+CD45RO+ cells and CD8+CD45RO+ cells with mSWAT $(n=14)$. Quantification of the number of cells per visual field was performed using the Hybrid Cell Count BZ$\mathrm{H} 4 \mathrm{C}$ analyzer software. Data were statistically analyzed using the Pearson correlation test (2-tailed). j) Results of multiplex chemokine bead assay using sera from peripheral and lesional blood $(n=14)$. Paired t-test. * $P<0.05$, ** $P<0.01$. 
a

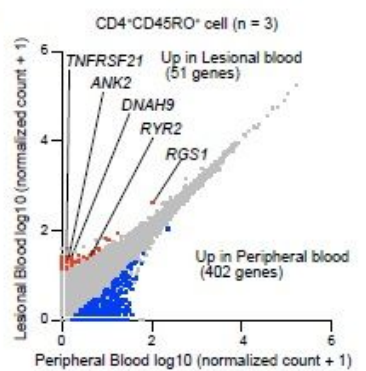

c

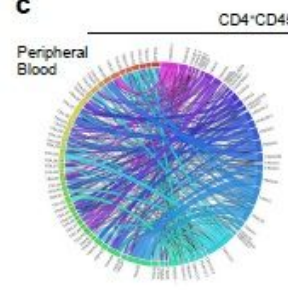

e

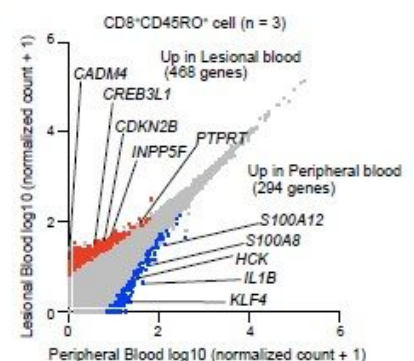

g

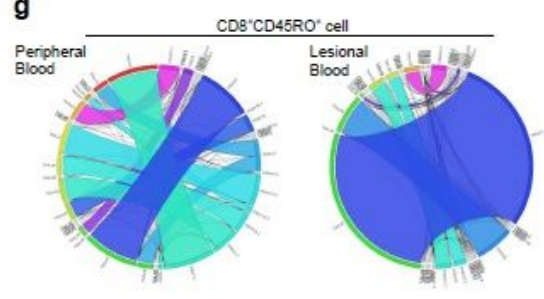

f b

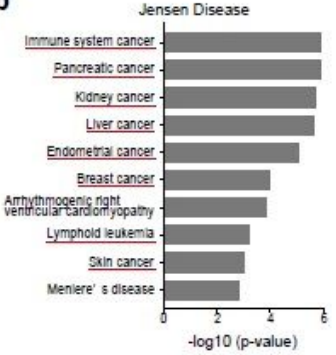

d

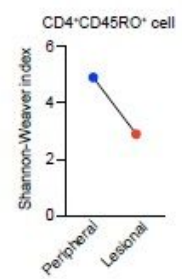

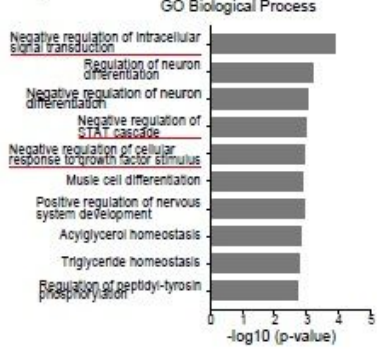

h

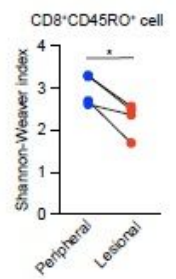

Figure 3

TCR repertoire of lesional blood is skewed, and expression of genes differs between lesional and peripheral blood. a) Gene expression analysis through RNA-seq in CD4+CD45RO+ T cells of peripheral and lesional blood from 3 patients (cases 12, 16, and 17). Scatter plots show the expression values of every annotated gene. Blue and red dots indicate significant upregulation of CD4+CD45RO+ T cells in peripheral and lesional blood, respectively. b) Ten top-ranked terms from the Jensen Diseases library of Enrichr for genes upregulated in CD4+CD45RO+ T cells of lesional blood. c) Circos plots of frequencies of $\mathrm{Va}$ and Ja gene usage and combinations of productive sequences in CD4+CD45RO+ T cells of peripheral and lesional blood. d) Diversity of the TCR repertoire in CD4+CD45RO+ T cells of peripheral and lesional blood was evaluated using the ShannonWeaver Index. e) RNA-seq in CD8+CD45RO+ T cells of peripheral and lesional blood from 3 patients (cases 4, 18, and 19). f) Ten top-ranked gene ontology terms of biologic processes for genes upregulated in CD8+CD45RO+ T cells of lesional blood. g) Circos plots of CD8+CD45RO+ T cells of peripheral and lesional blood. h) Shannon-Weaver Index of CD8+CD45RO+ T cells of peripheral and lesional blood. 

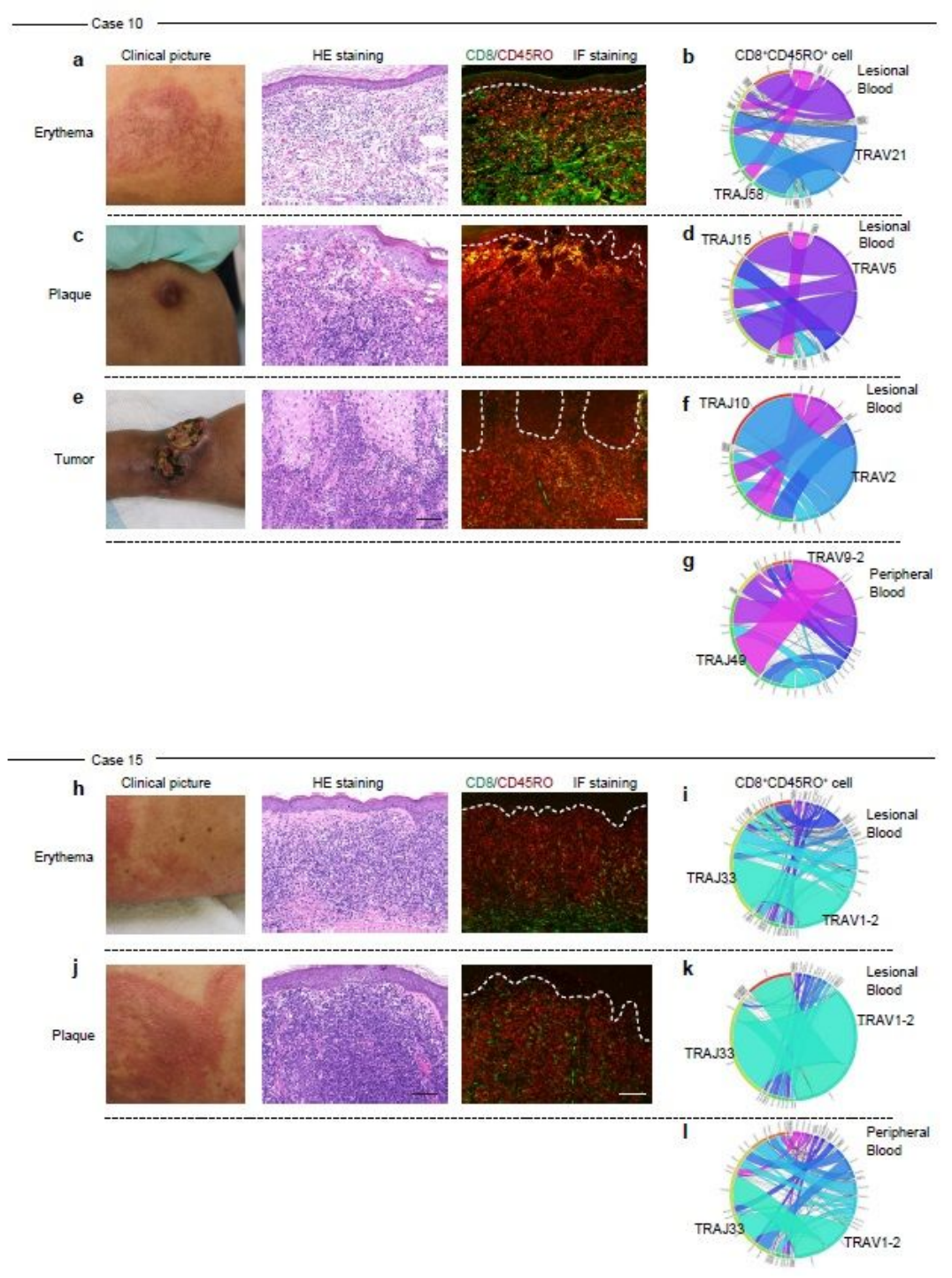

Figure 4

CD8+CD45RO+ TCR repertoire differ depending on the stage of MF.a, c, e) Hematoxylin and eosin (HE) staining and immunofluorescence (IF) staining of 3 types of skin lesions (erythema, plaque, and tumor) from case 10. CD8 (green) and CD45RO (red) are shown in the immunofluorescence staining image. The strongly green-stained areas are collagen, and red blood cells are stained with strong green and red. Scale bar $=100 \mathrm{~mm}$. b, d, f, g) Circos plots of frequencies of Va and Ja gene usage and combinations for CD8+CD45RO+ T cells in lesional blood from erythema, plaque, and tumor stages. The Circos plot at the bottom ( $\mathrm{g}$ ) is of cells obtained from peripheral blood. $\mathrm{h}-\mathrm{I})$ Erythema and plaque of the same skin lesion from case 15. Same experiment as shown in panels a - $\mathrm{g}$.

\section{Supplementary Files}

This is a list of supplementary files associated with this preprint. Click to download.

- 07042021SciReptable1.docx 